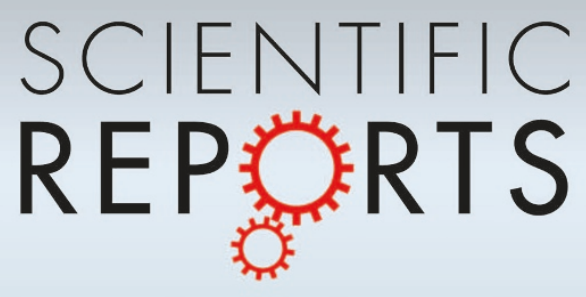

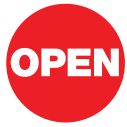

SUBJECT AREAS:

QUANTUM PHYSICS

BIOPHYSICS

STATISTICAL PHYSICS, THERMODYNAMICS AND NONLINEAR DYNAMICS

COMPUTATIONAL BIOLOGY

Received

10 May 2012

Accepted

16 July 2012

Published

13 August 2012

Correspondence and requests for materials should be addressed to A.A.-G. laspuru@ chemistry.harvard.

edu)

\title{
Finding low-energy conformations of lattice protein models by quantum annealing
}

\author{
Alejandro Perdomo-Ortiz' ${ }^{1}$ Neil Dickson², Marshall Drew-Brook², Geordie Rose² \& Alán Aspuru-Guzik' \\ 'Department of Chemistry and Chemical Biology, Harvard University, 12 Oxford Street, Cambridge, MA 02138, USA, ${ }^{2}$ D-Wave \\ Systems, Inc., 100-4401 Still Creek Drive, Burnaby, British Columbia V5C 6G9, Canada.
}

Lattice protein folding models are a cornerstone of computational biophysics. Although these models are a coarse grained representation, they provide useful insight into the energy landscape of natural proteins. Finding low-energy threedimensional structures is an intractable problem even in the simplest model, the Hydrophobic-Polar (HP) model. Description of protein-like properties are more accurately described by generalized models, such as the one proposed by Miyazawa and Jernigan (MJ), which explicitly take into account the unique interactions among all 20 amino acids. There is theoretical and experimental evidence of the advantage of solving classical optimization problems using quantum annealing over its classical analogue (simulated annealing). In this report, we present a benchmark implementation of quantum annealing for lattice protein folding problems (six different experiments up to 81 superconducting quantum bits). This first implementation of a biophysical problem paves the way towards studying optimization problems in biophysics and statistical mechanics using quantum devices.

$\mathrm{T}$ he search for more efficient optimization algorithms is an important endeavor with prevalence on many disciplines ranging from the social sciences to the physical and natural sciences. Belonging to the latter, the protein folding problem ${ }^{1-7}$ consists of finding the lowest free-energy configuration or, equivalently, the native structure of a protein given its amino-acid sequence. Knowing how proteins fold elucidate their three-dimensional structure-function relationship which is crucial to the understanding of enzymes and for the treatment of misfolded-protein diseases such as Alzheimer's, Huntington's, and Parkinson's disease. Due to the high computational cost of modeling proteins in atomistic detail ${ }^{8,9}$, coarse-grained descriptions of the protein folding problem, such as those found in lattice models, provide valuable insight about the folding mechanisms $s^{2,4-6,10}$.

Harnessing quantum-mechanical effects to speed up the solving of classical optimization problems is at the heart of quantum annealing algorithms $(\mathrm{QA})^{11-15}$. There is theoretical ${ }^{11,12,16-18}$ and experimental ${ }^{19}$ evidence of the advantage of solving classical optimization problems using $\mathrm{QA}^{11-14}$ over its classical analogue (simulated annealing $^{20}$ ). In QA, quantum mechanical tunneling allows for more efficient exploration of difficult potential energy landscapes such as that of classical spin-glass problems. In our implementation of lattice folding, quantum fluctuations (tunneling) occurs between states representing different model protein conformations or folds.

The theoretical challenge is to efficiently map the hard computational problem of interest (e.g., lattice folding) to a classical spin-glass Hamiltonian: such mapping requiring a polynomial number of quantum bits (qubits) with the size of the problem (protein length) is described elsewhere ${ }^{21}$. Here we present a new mapping which, due to its exponential scaling with problem size, is not intended for large instances. The proposed mapping employs very few qubits for small problem instances, making it ideal for this first experimental demonstration and implementation on current quantum devices ${ }^{22}$. A combination of the existing polynomial mapping ${ }^{21}$ and more advanced quantum devices would allow for the simulation of much larger instances of lattice folding and other related optimization problems.

Solving arbitrary problem instances requires a programmable quantum device to implement the corresponding classical Hamiltonian. We employ quantum annealing on the programmable device to obtain low-energy conformations of the protein model. We emphasize that nothing quantum mechanical is implied about the protein or its folding process; rather quantum fluctuations are a tool we use to solve the optimization problem.

The QA protocol performed here is also known as adiabatic quantum computation (AQC) $)^{17,23}$. Of all the quantum-computational models, AQC is perhaps the most naturally suited for studying and solving optimization problems ${ }^{1724}$. For the experiments presented here, the small finite temperature of the superconducting device is 
enough to make the process less coherent than the original formulation of AQC, where the theoretical limit of zero temperature and quasi-adiabaticity are usually assumed ${ }^{17,23}$. As we show in the discussion, numerical simulations including these unavoidable environmental effects accurately reproduce our experimental results.

Experimental implementations of QA or AQC are limited either by the number of qubits available in state-of-the-art quantum devices or by the programmability required to fulfill the problem specification. For example, the first realization of AQC was performed on a three-qubit NMR quantum device ${ }^{25}$ and newer NMR implementations involve four qubit experiments ${ }^{26}$. Other experimental realizations of spin systems have been based on measuring bulk magnetization properties of the systems in which there is no control over the individual spins and the couplings among them ${ }^{19,27,28}$. Quantum architectures using superconducting qubits ${ }^{29-36}$ offer promising device scalability while maintaining the ability to control individual qubits and the strength of their interaction couplings. During the preparation of this manuscript, an 84-qubit experimental determination of Ramsey numbers with quantum annealing was performed $^{37}$, underscoring the programmable capabilities of the device for problems with over 80 qubits. In this letter, we present a quantum annealing experimental implementation of lattice protein models with general (Miyazawa-Jernigan ${ }^{38}$ ) interactions among the amino acids. Even though the cases presented here still can be solved on a classical computer by exact enumeration (the six-amino-acid problem has only 40 possible configurations), it is remarkable that the device anneals to the ground state of a search space of $2^{81}$ possible computational outcomes. This study provides a proof-of-principle that optimization of biophysical problems such as protein folding can be studied using quantum mechanical devices.
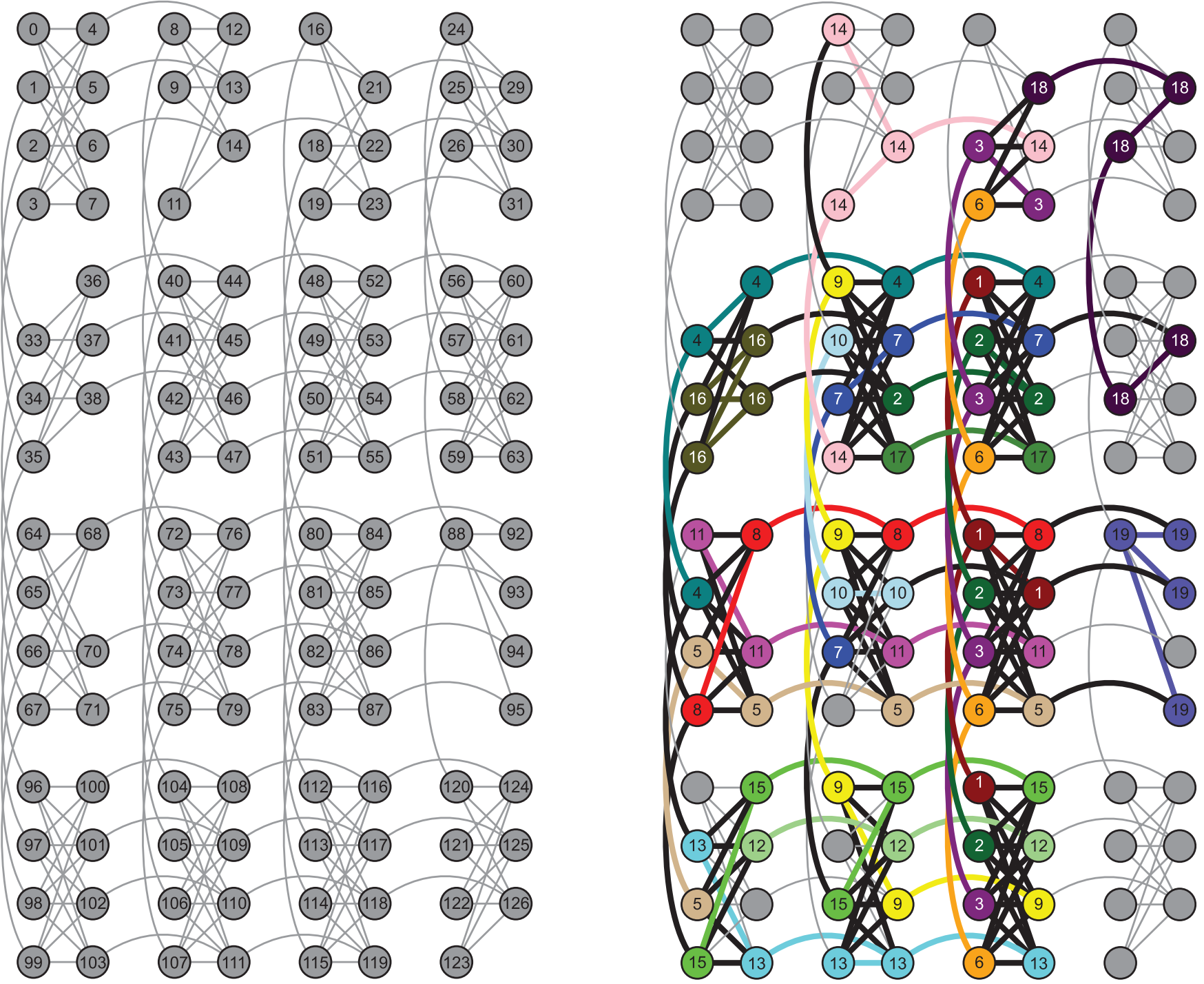

Figure $1 \mid$ Device architecture and qubit connectivity. The array of superconducting quantum bits is arranged in $4 \times 4$ unit cells that consist of 8 quantum bits each. Within a unit cell, each of the 4 qubits in the left-hand partition (LHP) connects to all 4 qubits in the right-hand partition (RHP), and vice versa. A qubit in the LHP (RHP) also connects to the corresponding qubit in the LHP (RHP) of the units cells above and below (to the left and right of) it. (a) Qubits are labeled from 0 to 127 and edges between qubits represent couplers with programmable coupling strengths. Grey qubits indicate the 115 usable qubits, while vacancies indicate qubits under calibration which were not used. The larger experiments (Experiments 1,2, and 4) were performed on this chip, while the three remaining smaller experiments were run on other chips with the same architecture. (b) Embedding and qubit connectivity for Experiment 4, coloring the 81 qubits used in the experiment. Nodes with the same color represent the same logical qubit from the original 19-qubit Ising-like Hamiltonian resulting from the energy function associated with Experiment 4 (see Supplementary material for details). This embedding aims to fulfill the arbitrary connectivity of the Ising expression and allows for the coupling of qubits that are not directly coupled in hardware. 


\section{Results}

The quantum hardware employed consists of 16 units of a recently characterized eightqubit unit cell ${ }^{22,39}$. Post-fabrication characterization determined that only 115 qubits out of the 128 qubit array can be reliably used for computation (see Fig. 1). The array of coupled superconducting flux qubits is, effectively, an artificial Ising spin system with programmable spin-spin couplings and transverse magnetic fields. It is designed to solve instances of the following (NPhard $^{40}$ ) classical optimization problem: Given a set of local longitudinal fields $\left\{h_{i}\right\}$ and an interaction matrix $\left\{J_{i j}\right\}$, find the assignment $\mathbf{s}^{*}=s_{1}^{*} s_{2}^{*} \cdots s_{N}^{*}$, that minimizes the objective function $E(\mathbf{s})$, where,

$$
E(\mathbf{s})=\sum_{1 \leq i \leq N} h_{i} s_{i}+\sum_{1 \leq i<j \leq N} J_{i j} s_{i} s_{j},
$$

$\left|h_{i}\right| \leq 1,\left|J_{i j}\right| \leq 1$, and $s_{i} \in\{+1,-1\}$.

Finding the optimal $s^{*}$ is equivalent to finding the ground state of the corresponding Ising classical Hamiltonian,

$$
H_{p}=\sum_{1 \leq i \leq N}^{N} h_{i} \sigma_{i}^{z}+\sum_{1 \leq i<j \leq N}^{N} J_{i j} \sigma_{i}^{z} \sigma_{j}^{z}
$$

where $\sigma_{i}^{z}$ are Pauli matrices acting on the $i$ th spin.

Experimentally, the time-dependent quantum Hamiltonian implemented in the superconductingqubit array is given by,

$$
H(\tau)=A(\tau) H_{b}+B(\tau) H_{p}, \quad \tau=t / t_{r u n},
$$

with $H_{b}=-\sum_{i} \sigma_{i}^{x}$ responsible for quantum tunneling among the localized classical states, which correspond to the eigenstates of $H_{p}$ (the computational basis). The time-dependent functions $A(\tau)$ and $B(\tau)$ are such that $A(0) \gg B(0)$ and $A(1) \ll B(1)$; in Fig. 2(b), we plot these functions as implemented in the experiment. $t_{\text {run }}$ denotes the time elapsed between the preparation of the initial state and the measurement.

QA exploits the adiabatic theorem of quantum mechanics, which states that a quantum system initialized in the ground state of a timedependent Hamiltonian remains in the instantaneous ground state, as long as it is driven sufficiently slowly. Since the ground state of $H_{p}$ encodes the solution to the optimization problem, the idea behind QA is to adiabatically prepare this ground state by initializing the quantum system in the easy-to-prepare ground state of $H_{b}$, which corresponds to a superposition of all $2^{N}$ states of the computational basis. The system is driven slowly to the problem Hamiltonian, $H(\tau=1) \approx H_{p}$. Deviations from the ground-state are expected due to deviations from adiabaticity, as well as thermal noise and imperfections in the implementation of the Hamiltonian.

The first challenge of the experimental implementation is to map the computational problem of interest into the binary quadratic expression (Eq. 2), which we outline next. In lattice folding, the sequence of amino acids defining the protein is viewed as a sequence of beads (amino acids) connected by strings (peptide bonds). This bead chain occupies points on a two- or three-dimensional lattice. A valid configuration is a self-avoiding walk on the lattice and its energy (a)

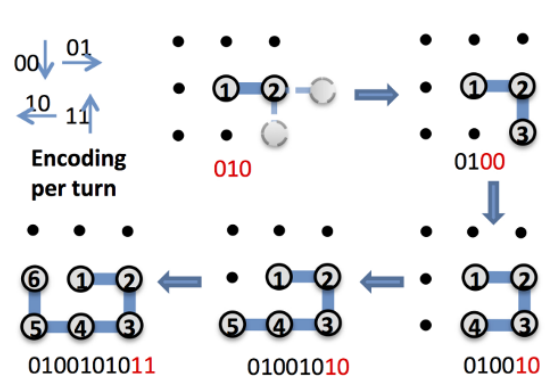

(c)

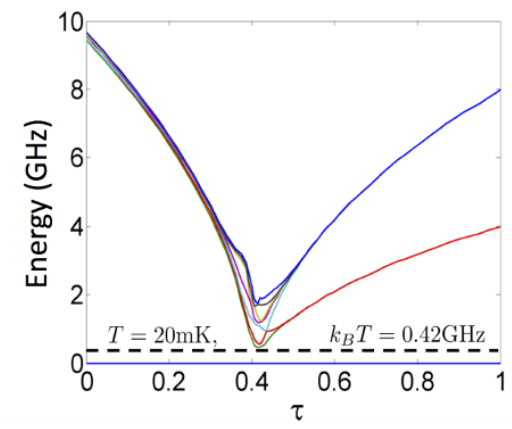

(b)

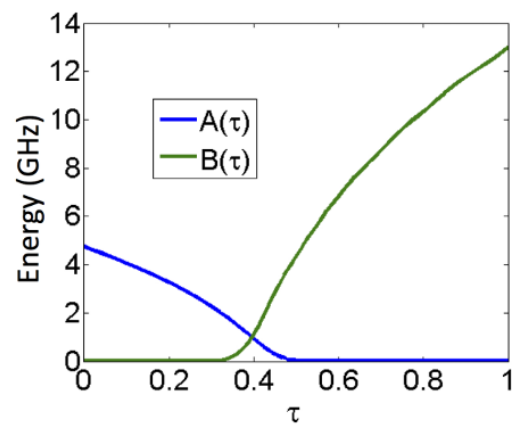

(d)

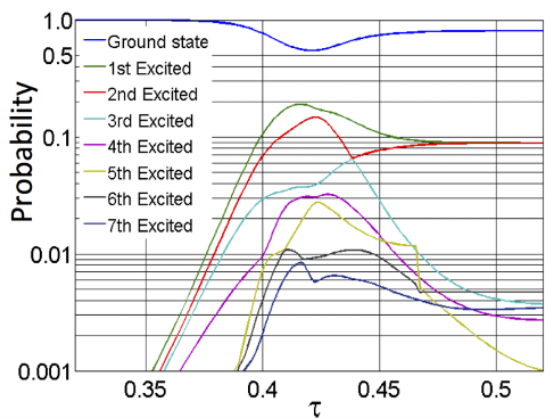

Figure $2 \mid$ Lattice folding mapping for quantum annealing. (a) Step-by-step construction of the binary representation of lattice protein. Two qubits per bond are needed and the bond directions are denoted as "00" (downwards), "01" (rightwards), "10" (leftwards), and "11" (upwards). The example shows one of the possible folds of an arbitrary six-amino-acid sequence. Any possible $\mathrm{N}$-amino-acid fold can be represented by a string of variables $010 q_{1} q_{2} q_{3} \cdots q_{\ell-1} q_{\ell}$ with $\ell=2 N-5$. (b)Time-dependence of the $A(\tau)$ and $B(\tau)$ functions, where $\tau=t / t_{\text {run }}$ with $t_{\text {run }}=148 \mu s$, (c) time-dependent spectrum obtained through numerical diagonalization, and (d) Bloch-Redfield simulations showing the time-dependent population in the first eight instantaneous eigenstates of the experimentally implemented 8-qubit Hamiltonian (Eq. 3) with $H_{p}$ from Eq. S18 in the Supplementary material. In panel (c), for each instantaneous eigenenergy curve we have subtracted the energy of the ground state, effectively plotting the gap of the seven-lowest-excited states with respect to the ground state (represented by the baseline at zero-energy). As a reference, we show the energy with the device temperature, which is comparable to the minimum gap between the ground and first excited state. In panel (d), populations are ordered in energy from top (ground state) to bottom. Although $\tau=t / t_{\text {run }}$ runs from 0 to 1 , we show the region where most of the population changes occur. As expected, this is in the proximity of the minimum gap between the ground and first excited state around $\tau \sim 0.4$ [see panel(c)]. 
is calculated from the sum of interaction energies between nearest non-bonded neighbors on the lattice. By the thermodynamic hypothesis of protein folding ${ }^{41}$, the global minimum of the free-energy function is conjectured to be the native functional conformation of the protein.

Finding low-energy three-dimensional structures is an intractable problem $^{42-44}$. The hydrophobic-polar (HP) model is one of the simplest possible models for lattice folding ${ }^{45}$. In this model, the amino acids are classified into two groups, hydrophobic $(\mathrm{H})$ and polar $(\mathrm{P})$. Even in this simplest model, exhaustive search of all possible global minima is limited to sequences in the tens of amino acids ${ }^{46}$. To describe real protein energy landscapes a more elaborate description needs to be considered, such as the Mijazawa-Jernigan (MJ) model ${ }^{38}$ which assigns the interaction energies for pairwise interactions among all twenty amino acids. The formulation we used is general enough to take into account arbitrary interaction matrices for lattice models in two and three dimensions. In particular, we solved a MJ model in 2D, the six-amino-acid sequence of Proline-Serine-ValineLysine-Methionine-Alanine (PSVKMA in the one-letter amino-acid sequence notation). We solved the problem under two different experimental schemes (see Schemes 2 and 3 in Fig. 3), each requiring a different number of resources. Solving the problem in one proposed experimental realization (Scheme 1) requires more resources than the number of qubits available (115 qubits) in the device. Scheme 2 and 3 are examples of the divide-and-conquer strategy, in which one partitions the problem in smaller instances and combines the independent set of results, thereby obtaining the same solution for the intractable problem. In the Supplementary Information section, we complement these four MJ related experiments with two small tetrapeptide instances (effectively HP model instances) for a total of six different problem Hamiltonians. We used the largest of these two instances (an 8 qubit experiment) for direct theoretical simulation of the annealing dynamics of the device. The results from our experiment and the theoretical model, which does not use any adjustable parameters (all are extracted experimentally from the device), are in excellent agreement (see Supplementary Fig. S2 online).

To represent each of the possible $\mathrm{N}$-amino-acid configurations (folds) in the lattice, we encode the direction of each successive bond between amino acids; thus, for every $N$-bead sequence we need to specify $N-1$ turns corresponding to the number of bonds. For the case of a two dimensional lattice, a bond can take any of four possible directions; therefore, two bits per bond are required to uniquely determine a direction. More specifically, if a bond points upwards, we write " 11 ". If it points downwards, leftwards or rightwards, we (a)

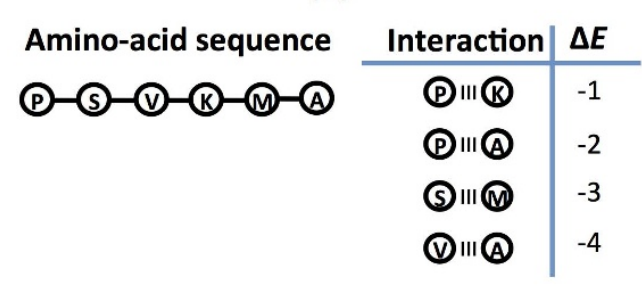

(b)

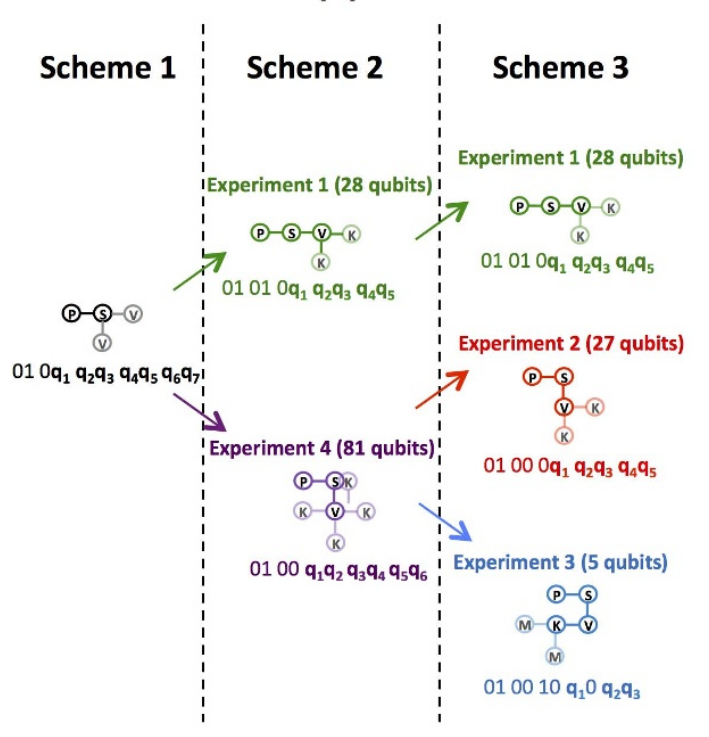

(c)

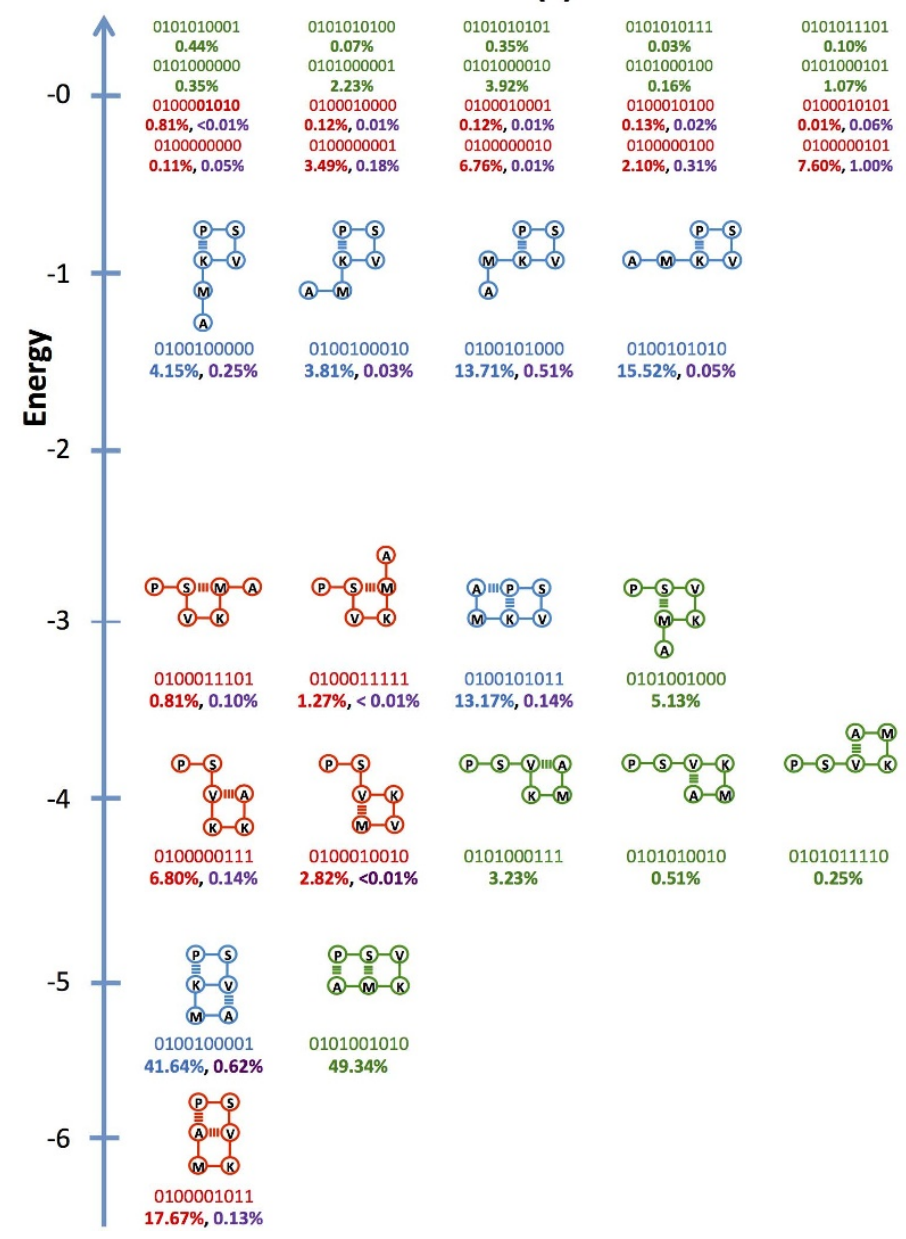

Figure 3 Experimental realizations. (a) Representation of the six-amino-acid sequence, Proline-Serine-Valine-Lysine-Methionine-Alanine with its

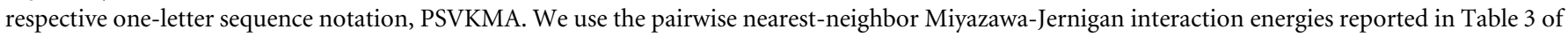

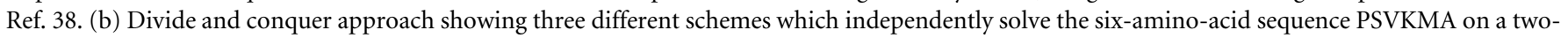

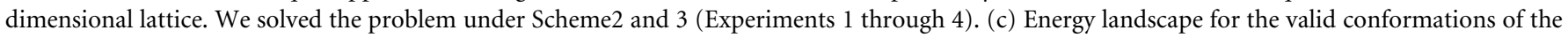
PSVKMA sequence. Results of the experimentally-measured probability outcomes are given as color-coded percentages according to each of the experimental realizations described in panel (b). Percentages for states with energy greater than zero are $32.70 \%$, $59.88 \%$, $8.00 \%$, and $95.97 \%$ for Experiments 1 through 4, respectively. 
write " 00 ", " 10 ", or " 01 " respectively. Fixing the direction of the first bond reduces the description of any $N$-bead fold to $\ell=2(N-2)$ binary variables, without loss of generality. As shown in Fig. 2(a), in the absence of external constraints other than those imposed by the primary amino-acid sequence (see Supplementary Information for an example with external constraints), we can fix the third binary variable to " 0 ", forcing the third amino acid to go either straight or downward and reducing the number of needed variables to $\ell=2 N-5$. This constraint reduces the solution space by removing conformations which are degenerate due to rotational symmetry. Thus, a particular fold is uniquely defined by,

$$
\boldsymbol{q}=\underbrace{01}_{\text {turn } 1} \underbrace{0 q_{1}}_{\text {turn } 2} \underbrace{q_{2} q_{3}}_{\text {turn } 3} \cdots \underbrace{q_{2 N-6} q_{2 N-5}}_{\text {turn }(N-1)}
$$

An example of this encoding for a six-amino-acid sequence is represented in Fig. 2(a).

Using this mapping to translate between the amino-acid chain in the lattice and the $2(N-1)$ string of bits, we constructed the energy function $E(\boldsymbol{q})$ in which $\boldsymbol{q}$ denotes the remaining $2 N-5$ binary variables. Additionally, we penalized folds which exhibit two amino acids on top of each other, to favor self-avoiding walk configurations. The energy penalty chosen for each problem was sufficient to push the energy of invalid folds outside of the energy range of valid configurations (those with $E \leq 0$ ). Finally, we took into account the interaction energy among the different amino acids. A detailed construction of our energy function for the general case of $N$ amino acids with arbitrary interactions is given elsewhere.

The experiment consists of the following steps: a) construction of the energy function to be minimized in terms of the turn encoding; $b$ ) reduction of the energy expression to a two-body Hamiltonian; and finally, c) embedding in the device. These last two steps need additional resources as explained below. We will focus on the simplest example (Experiment 3, Fig. 3) to show the procedure in detail. The embeddings for the other five experiments are provided in the Supplementary material. The energy function for Experiment 3, containing the contributions due to on-site penalties for overlapping amino acids, and pairwise interactions between amino acids is,

$$
E(\boldsymbol{q}) \equiv E_{\text {exp } 3}^{\text {cubic }}=-1-4 q_{3}+9 q_{1} q_{3}+9 q_{2} q_{3}-16 q_{1} q_{2} q_{3}
$$

where $q_{1} 0\left(q_{2} q_{3}\right)$ encodes the orientation of the fourth (fifth) bond (see Fig. 3). From Eq. 5 one can verify by substitution that the eight possible three-bit-variable assignments provide the desired energy landscape: the six conformations with $E \leq 0$ shown in blue in Fig. 3 .

Eq. 5 describes the energy landscape of configurations but it is not quite ready for the device. Experimentally, we can specify up to twobody spin interactions, $\sigma_{i}^{z} \sigma_{j}^{z}$, and therefore, we need to convert this cubic energy function (Eq. 5) into a quadratic form resembling Eq. 1 (see Supplementary Information for details). The resulting expression is

$$
\begin{aligned}
H_{p}^{\text {unembedded }}= & \left(7 \sigma_{1}^{z}+9 \sigma_{2}^{z}+8 \sigma_{3}^{z}-20 \sigma_{4}^{z}+9 \sigma_{1}^{z} \sigma_{3}^{z}+9 \sigma_{2}^{z} \sigma_{3}^{z}\right. \\
& \left.-16 \sigma_{1}^{z} \sigma_{4}^{z}-18 \sigma_{2}^{z} \sigma_{4}^{z}-18 \sigma_{3}^{z} \sigma_{4}^{z}\right) / 4
\end{aligned}
$$

where the original binary variables and spin operators are related by $q_{i} \rightarrow\left(1-\sigma_{i}^{z}\right) / 2$. Experimental measurements of $\sigma_{i}^{z}$ yield $s_{i}=+1$ $\left(s_{i}=-1\right)$ corresponding to $q_{i}=0\left(q_{i}=1\right)$. Since $q_{i}=\left(1-s_{i}\right) / 2$, measurement of $s_{1}, s_{2}$, and $s_{3}$ allows us to reconstruct the bit string $q_{1} 0 q_{2} q_{3}$ which encodes the desired fold.

One ancilla variable was added during the transformation of the three-variable cubic Hamiltonian into this quadratic four-variable expression. The meaning of the original variables $s_{1}, s_{2}$, and $s_{3}$ remains the same, allowing for the reconstruction of the folds. The energy of this four-variable expression will not change as long as the measurements of $\sigma_{1}^{z}$ through $\sigma_{4}^{z}$ result in values for $q_{1} q_{2} q_{3} q_{4}$ satisfying $q_{4}=q_{2} q_{3}$. This transformation ensures an energy penalty whenever this condition is violated.

The architecture of the chip lacks sufficient connectivity between the superconducting rings for a one-to-one assignment of variables to qubits (see Fig. 4). To satisfy the connectivity requirements of the four-variable energy function, the couplings of one of the most connected variables, $q_{4}$, were fulfilled by duplicating this variable inside the device such that $q_{4} \rightarrow q_{4}$ and $q_{4^{\prime}}$. In the form of Eq. 2 the final expression representing the energy function of Experiment 3 is given by,

$$
\begin{aligned}
H_{p}= & \left(7 \sigma_{1}^{z}+9 \sigma_{2}^{z}+8 \sigma_{3}^{z}-2 \sigma_{4}^{z}-18 \sigma_{4^{\prime}}^{z}+9 \sigma_{1}^{z} \sigma_{3}^{z}+9 \sigma_{2}^{z} \sigma_{3}^{z}-16 \sigma_{1}^{z} \sigma_{4}^{z}\right. \\
& \left.-18 \sigma_{2}^{z} \sigma_{4}^{z}-18 \sigma_{3}^{z} \sigma_{4^{\prime}}^{z}-36 \sigma_{4}^{z} \sigma_{4^{\prime}}^{z}\right) / 36 .
\end{aligned}
$$

This expression satisfies all requirements for the problem Hamiltonian (Eq. 3), the completion of which allows for the measurement of the energetic minimum conformation of this small peptide instance. The embedding of Eq. 7 into the hardware is shown in Fig. 4 , where we label the five qubits used, $q_{1}, q_{2}, q_{3}, q_{4}$, and $q_{4^{\prime}}$. Since we want the two qubits representing $q_{4}$ to end up with the same value, we apply the maximum ferromagnetic coupling $(J=-1)$ between them, which adds a penalty whenever this equality is violated (last term in Eq. 7). These maximum couplings are indicated in Fig. 4 by heavy lines. The thinner lines show the remaining couplings used to realize the quadratic terms in Eq. 7, color coded according to the sign of the interaction and its thickness representing their strength. Note that every quadratic term in Eq. 7 has a corresponding coupler. Hereafter, we will denote the outcome of the five-qubit measurements as $\boldsymbol{q}_{\exp 3}=010010 q_{1} 0 q_{2} q_{3} \mid q_{4} q_{4^{\prime}}$, with $q_{i}=0\left(q_{i}=1\right)$ whenever $s_{i}=1\left(s_{i}=-1\right)$. Notice that only the bits preceding the divider character $\mid$ contain physical information. These are the ones shown under each of the protein fold drawings associated with Experiment 3 (see Fig. 3).

Similar embedding procedures to the one previously described were used for the larger experiments. For example, in Experiment 1 , only 5 qubits define solutions of the computational problem. We
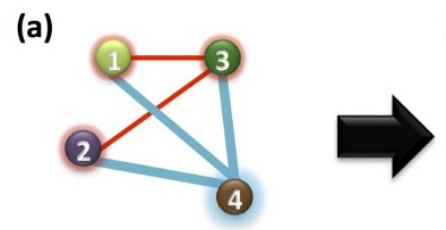

(b)
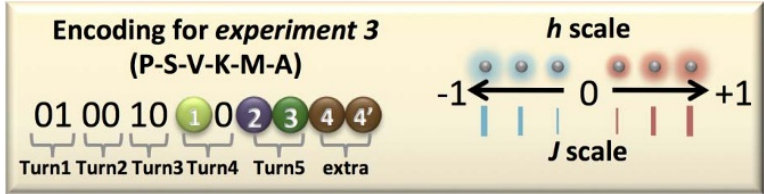

Figure $4 \mid$ Embedding problem instances into hardware. Graph representations of (a) the four-qubit unembedded energy function (Eq. 6) and (b) the five-qubit expression (Eq. 7) as was embedded into the quantum hardware. In graphs (a) and (b), each node denotes a qubit and the color and extent of its glow denotes the sign and strength of its corresponding longitudinal field, $h_{i}$. The edges represent the interaction couplings, $J_{i j}$, where color indicates sign and thickness indicates magnitude. Since we want the two qubits representing $q_{4}\left(q_{4}\right.$ and $\left.q_{4^{\prime}}\right)$ to end up with the same value, we apply the maximum ferromagnetic coupling $(J=-1)$ between them, which adds a penalty whenever this equality is violated. These maximum couplings are indicated in the figure by heavy lines. For the case of Experiment 3, the reconstruction of the binary bit stings representing the folds in Fig. 3, from the five-qubit experimental measurements can be recovered by $\boldsymbol{q}_{\exp 3}=$ $010010 q_{1} 0 q_{2} q_{3} \mid q_{4} q_{4^{\prime}}$, with $q_{i}=0\left(q_{i}=1\right)$ whenever $s_{i}=1\left(s_{i}=-1\right)$. 
needed 5 auxiliary qubits to transform the expression with 5-body interactions into an expression with only 2-body interactions. Embedding of this final expression required an additional of 18 qubits to satisfy the hardware connectivity requirements, for a total of 28 qubits. Table $\mathrm{S} 1$ in the Supplementary material summarizes the number of qubits required in each step through to the final experimental realizations.

\section{Discussion}

Even though the quantum device follows a quantum annealing protocol, the odds of measuring the ground state are not necessarily high. For example, in the 81 qubit experiment, only 13 out of 10,000 measurements yielded the desired solution. We attribute these low-percentages to the analog nature of the device and to precision limitations in the real values of the local fields and couplings among the qubits in the experimental setup. When compared to other problem implementations, physical problems such as lattice folding lack the structure of the Ramsey number problem ${ }^{37}$. In the lattice folding problem implemented here, the parameters defining the problem instances are arbitrary and do not fall into certain integral distinct values as in the case of the Ramsey number experiment, making precision issues more pronounced in our implementation.

To gain insights into the dynamics and evolution of the quantum system, we numerically simulated the superconducting array with a Bloch-Redfield model of the 8-qubit experiment (see Supplementary material) which takes into account thermal fluctuations in the states due to the finite temperature $(20 \mathrm{mK})$ of the quantum device. For this 8 -qubit experiment, the simulation predicted a ground state probability of $80.7 \%$, in excellent agreement with the experimentally observed value $(80.3 \%)$. It is important to note that no adjustable parameters were used in our simulations to fit the data and all the parameters correspond to values measured directly from the quantum device. More details about the numerical simulations can be found in the Supplementary Information.

As seen in Fig. 2(c), the temperature of the device is comparable with the minimum gap of the eight-qubit Hamiltonian. Therefore, we expect stronger excitation/relaxation near the gap closing, $\tau \approx 0.6$, due to exchange of energy with the environment, when compared to the other regimes of the annealing schedule where the gap is much larger than $k_{B} T$. In the absence of environment (a fully coherent process), our simulations indicate that that the success probability would be $100 \%$, within numerical error. Fig. 2(d) shows that for the simulations at $20 \mathrm{mK}$, the probability in the ground state goes down to $\sim 55 \%$, but the same fluctuations make the system relax back to the ground state, yielding $\tan 80.27 \%$ success probability. This is due to the advantageous natural tendency of the system to approach a thermal equilibrium which favors the ground state after crossing the minimum energy gap. As previously discussed in similar numerical simulations of quantum annealing algorithms ${ }^{47}$, strong coupling to the bath and non-Markovianity would require going beyond the Bloch-Redfield model $^{48}$, but the agreement between experimental and simulated results support the validity of the quantum mechanical model used to describe the device. Previously reported temperature dependence predictions for the tunneling rate on the same qubits $^{22}$ and excellent agreement with the same level of theory used here reinforce the validity of our simulations for this 8-qubit instances.

We present the first quantum-mechanical implementation of lattice protein models using a programmable quantum device. We were able to encode and to solve the global minima solution for a small tetrapeptide and hexapeptide chain under several experimental schemes involving 5 and 8 qubits for the four-amino-acid sequence (Hydrophobic-Polar model) and 5, 27, 28, and 81 qubits experiments for the six-amino-acid sequence under the Miyazawa-Jernigan model for general pairwise interactions. For the experiment with 8 qubits, we simulated the dynamics of the quantum device with a
Redfield equation with no adjustable parameters, obtaining excellent agreement with experiment. Since the quantum annealing algorithm not only finds the ground state but also the low-lying excited states, it provides information about the relevant minimum energy compact structures of protein sequences ${ }^{49}$ and it is useful to evaluate designability and stability such as that found in natural protein sequences, where the global minimum of free energy is well separated in energy from other misfolded states ${ }^{41}$. The approach employed here can be extended to treat other problems in biophysics and statistical mechanics such as molecular recognition, protein design, and sequence alignment $^{50}$.

1. Šali, A., Shakhnovich, E. \& Karplus, M. How does a protein fold? Nature 369, 248-251 (1994).

2. Pande, V. S. Simple theory of protein folding kinetics. Phys. Rev. Lett. 105, 198101 (2010).

3. Dill, K. A., Ozkan, S. B., Shell, M. S. \& Weikl, T. R. The protein folding problem. Ann. Rev. Biophys. 37, 289-316 (2008).

4. Mirny, L. \& Shakhnovich, E. Protein folding theory: from lattice to all-atom models. Annu. Rev. Biophys. Bio. 30, 361-396 (2001).

5. Pande, V. S., Grosberg, A. Y. \& Tanaka, T. Heteropolymer freezing and design: Towards physical models of protein folding. Rev. Mod. Phys. 72, 259 (2000).

6. Kolinski, A. \& Skolnick, J. Lattice Models of Protein Folding, Dynamics and Thermodynamics (Chapman \& Hall, 1996).

7. Shakhnovich, E. I. Proteins with selected sequences fold into unique native conformation. Phys. Rev. Lett. 72, 3907 (1994).

8. Bohannon, J. Distributed computing: Grassroots supercomputing. Science 308, 810 (2005).

9. Shaw, D. E. et al. Atomic-Level characterization of the structural dynamics of proteins. Science 330, 341-346 (2010).

10. Li, M. S. et al. Factors governing fibrillogenesis of polypeptide chains revealed by lattice models. Phys. Rev. Lett. 105, 218101 (2010).

11. Finnila, A. B., Gomez, M. A., Sebenik, C., Stenson, C. \& Doll, J. D. Quantum annealing: A new method for minimizing multidimensional functions. Chem. Phys. Lett. 219, 343-348 (1994).

12. Kadowaki, T. \& Nishimori, H. Quantum annealing in the transverse ising model. Phys. Rev. E. 58, 5355 (1998).

13. Santoro, G. E. \& Tosatti, E. Optimization using quantum mechanics: quantum annealing through adiabatic evolution. J. Phys. A. 39, R393-R431 (2006).

14. Das, A. \& Chakrabarti, B. K. Colloquium: Quantum annealing and analog quantum computation. Rev. Mod. Phys. 80, 1061-1081 (2008).

15. Ray, P., Chakrabarti, B. K. \& Chakrabarti, A. Sherrington-Kirkpatrick model in a transverse field: Absence of replica symmetry breaking due to quantum fluctuations. Phys. Rev. B 39, 11828-11832 (1989).

16. Amara, P., Hsu, D. \& Straub, J. E. Global energy minimum searches using an approximate solution of the imaginary time schroedinger equation. J. Phys. Chem. 97, 6715-6721 (1993).

17. Farhi, E. et al. A quantum adiabatic evolution algorithm applied to random instances of an NP-Complete problem. Science 292, 472-475 (2001).

18. Santoro, G., Martonák, R., Tosatti, E. \& Car, R. Theory of quantum annealing of an ising spin glass. Science 295, 24272430 (2002).

19. Brooke, J., Bitko, D., Rosenbaum, T. F. \& Aeppli, G. Quantum annealing of a disordered magnet. Science 284, 779-781 (1999).

20. Kirkpatrick, S., Gelatt, C. D. \& Vecchi, M. P. Optimization by simulated annealing. Science 220, 671-680 (1983).

21. Perdomo, A., Truncik, C., Tubert-Brohman, I., Rose, G. \& Aspuru-Guzik, A. Construction of model hamiltonians for adiabatic quantum computation and its application to finding low-energy conformations of lattice protein models. Phys. Rev. A 78, 012320-15 (2008).

22. Johnson, M. W. et al. Quantum annealing with manufactured spins. Nature $\mathbf{4 7 3}$, 194-198 (2011).

23. Farhi, E., Goldstone, J., Gutmann, S. \& Sipser, M. Quantum computation by adiabatic evolution. arXiv:quant-ph/0001106 (2000).

24. Hogg, T. Adiabatic quantum computing for random satisfiability problems. Phys. Rev. A. 67, 022314 (2003)

25. Steffen, M., van Dam, W., Hogg, T., Breyta, G. \& Chuang, I. Experimental implementation of an adiabatic quantum optimization algorithm. Phys. Rev. Lett. 90, 067903 (2003).

26. Xu, N. et al. Quantum factorization of 143 on a dipolar-coupling nmr system. arXiv:1111.3726v1 (2011).

27. Ancona-Torres, C., Silevitch, D. M., Aeppli, G. \& Rosenbaum, T. F. Quantum and classical glass transitions in LiHo_xY_1-xF_4. Phys. Rev. Lett. 101, 057201 (2008).

28. Wernsdorfer, W. Molecular nanomagnets: towards molecular spintronics. Int. J. Nanotechnol. 7, 497-522 (2010).

29. Vion, D. et al. Manipulating the quantum state of an electrical circuit. Science 296, 886-889 (2002)

30. You, J. Q. \& Nori, F. Superconducting circuits and quantum information. Phys. Today. 58, 42-47 (2005). 
31. Lupascu, A. et al. Quantum non-demolition measurement of a superconducting two-level system. Nat. Phys. 3, 119-125 (2007).

32. Hofheinz, M. et al. Synthesizing arbitrary quantum states in a superconducting resonator. Nature 459, 546-549 (2009).

33. DiCarlo, L. et al. Demonstration of two-qubit algorithms with a superconducting quantum processor. Nature 460, 240-244 (2009).

34. Neeley, M. et al. Generation of three-qubit entangled states using superconducting phase qubits. Nature 467, 570-573 (2010)

35. DiCarlo, L. et al. Preparation and measurement of three-qubit entanglement in a superconducting circuit. Nature 467, 574-578 (2010).

36. Kaminsky, W. M., Lloyd, S. \& Orlando, T. P. Scalable superconducting architecture for adiabatic quantum computation. arXiv:quant-ph/0403090 (2004).

37. Bian, Z., Chudak, F., Macready, W. G., Clark, L. \& Gaitan, F. Experimental determination of ramsey numbers with quantum annealing. arXiv:1201.1842v2 (2012).

38. Miyazawa, S. \& Jernigan, R. L. Residue-residue potentials with a favorable contact pair term and an unfavorable high packing density term, for simulation and threading. J. Mol. Biol. 256, 623-644 (1996).

39. Harris, R. et al. Experimental investigation of an eight-qubit unit cell in a superconducting optimization processor. Phys. Rev. B. 82, 024511 (2010).

40. Barahona, F. On the computational complexity of ising spin glass models. J. Phys. A: Math. Gen. 15, 3241-3253 (1982).

41. Anfinsen, C. B. Principles that govern the folding of protein chains. Science 181, 223-230 (1973).

42. Berger, B. \& Leighton, T. Protein folding in the hydrophobic-hydrophilic (HP) model is NP-complete. J. Comput. Biol. 5, 27-40 (SPR 1998).

43. Crescenzi, P., Goldman, D., Papadimitriou, C., Piccolboni, A. \& Yannakakis, M. On the complexity of protein folding. J. Comput. Biol. 5, 597-603 (1998).

44. Hart, W. E. \& Istrail, S. Robust proofs of NP-Hardness for protein folding: General lattices and energy potentials. J. Comput. Biol. 4, 1-22 (1997).

45. Lau, K. F. \& Dill, K. A. A lattice statistical-mechanics model of the conformational and sequence-spaces of proteins. Macromolecules. 22, 3986-3997 (1989).

46. Yue, K. \& Dill, K. A. Forces of tertiary structural organization in globular proteins. Proc. Natl. Acad. Sci. USA. 92, 146-150 (1995).

47. Amin, M. H. S., Truncik, C. J. S. \& Averin, D. V. Role of single-qubit decoherence time in adiabatic quantum computation. Phys. Rev. A 80, 022303 (2009).
48. Amin, M. H. S., Averin, D. V. \& Nesteroff, J. A. Decoherence in adiabatic quantum computation. Phys. Rev. A 79, 022107 (2009).

49. Camacho, C. J. \& Thirumalai, D. Minimum energy compact structures of random sequences of heteropolymers. Phys. Rev. Lett. 71, 2505-2508 (1993).

50. Hartmann, A. K. \& Rieger, H. New Optimization Algorithms in Physics (Wiley-VCH, 2004)

\section{Acknowledgements}

This work was supported by NSF CCI center, "Quantum Information for Quantum Chemistry(QIQC)”, Award number CHE-1037992. The authors thank Sergio Boixo Mohammad Amin, and Ryan Babbush for helpful discussions and revisions of the manuscript.

\section{Author contributions}

A.P-O and A.A-G developed the theoretical constructs and designed the experiments. M.D-B, and G.R ran the experiments. N.D ran the Bloch-Redfield numerical simulations. A.P-O, N.D, and A.A-G wrote the manuscript and the Supplementary Information. All the authors revised the manuscript.

\section{Additional information}

Supplementary Information accompanies this paper at http://www.nature.com/ scientificreports

Competing financial interests: The authors declare no competing financial interests.

License: This work is licensed under a Creative Common

Attribution-NonCommercial-NoDerivative Works 3.0 Unported License. To view a copy of this license, visit http://creativecommons.org/licenses/by-nc-nd/3.0/

How to cite this article: Perdomo-Ortiz, A., Dickson, N., Drew-Brook, M., Rose, G. \& Aspuru-Guzik, A. Finding low-energy conformations of lattice protein models by quantum annealing. Sci. Rep. 2, 571; DOI:10.1038/srep00571 (2012). 